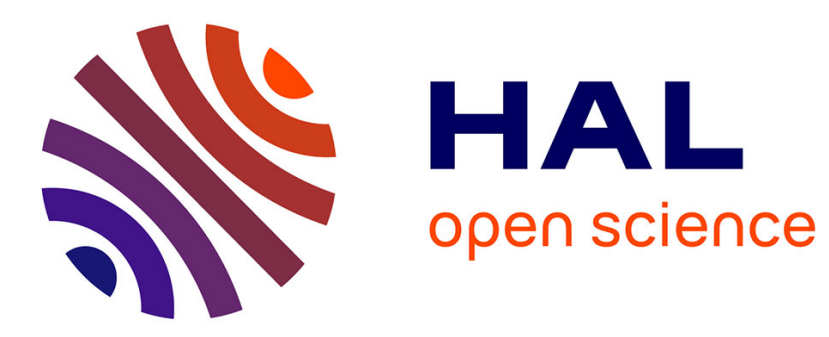

\title{
Transient pores in vesicles
}

Erdem Karatekin, Olivier Sandre, Françoise Brochard-Wyart

\section{To cite this version:}

Erdem Karatekin, Olivier Sandre, Françoise Brochard-Wyart. Transient pores in vesicles. Polymer international, 2003, 52 (4), pp.486-493. 10.1002/pi.1007 . hal-02168780

\section{HAL Id: hal-02168780 \\ https://hal.science/hal-02168780}

Submitted on 5 Aug 2019

HAL is a multi-disciplinary open access archive for the deposit and dissemination of scientific research documents, whether they are published or not. The documents may come from teaching and research institutions in France or abroad, or from public or private research centers.
L'archive ouverte pluridisciplinaire HAL, est destinée au dépôt et à la diffusion de documents scientifiques de niveau recherche, publiés ou non, émanant des établissements d'enseignement et de recherche français ou étrangers, des laboratoires publics ou privés. 


\title{
Transient pores in vesicles ${ }^{\dagger}$
}

\author{
Erdem Karatekin, ${ }^{1 \ddagger}$ Olivier Sandre ${ }^{2}$ and Françoise Brochard-Wyart ${ }^{1 *}$ \\ ${ }^{1}$ Institut Curie, Section de Recherche, Laboratoire PCC/UMR 168, 11, rue Pierre et Marie Curie, 75231 Paris Cedex 05, France \\ ${ }^{2}$ Laboratoire Liquides Ioniques et Interfaces Chargées UMR 7612 CNRS, Université Paris 6, 4 Place Jussieu, Case 63, 75252 Paris Cedex \\ 05, France
}

\begin{abstract}
We present our observations of transient pores in giant unilamellar vesicles, placed under tension, by optical illumination. When the membrane tension reached a certain level, transient pores appeared. Pore opening is driven by the membrane tension, $\sigma$, and its closure by the pore's line tension, $\mathcal{T}$. By use of viscous mixtures of glycerol and water, we slowed down the leak out of the inner liquid in the presence of a pore. This allowed pores to reach large sizes (a few micrometres) and last at least a few seconds so that they could be visualized by fluorescence videomicroscopy. Line tension was inferred from the measurements of the closure velocity of the pores. By addition of cholesterol, which increased $\mathcal{T}$ (reducing pore lifetimes), or of surfactants, which decreased $\mathcal{T}$ (increasing pore lifetimes), we demonstrate how $\mathcal{T}$, and consequently pore lifetimes, can be controlled over nearly two orders of magnitude. Addition of surfactants also has a dramatic effect on vesicle fusion. We discuss how our results can be extended to less viscous aqueous solutions which are more relevant for liposomal drug delivery formulations.

Author manuscript of proceedings paper of colloquium "Formula III: New Concepts and Strategies in Formulation: from Laboratory to Industry", Montpellier, Hérault, France, 13-16 October 2001
\end{abstract}

Keywords: Giant vesicles; line tension; DOPC; cholesterol; pores in membranes

\section{INTRODUCTION}

Liposomes are emerging as promising drug delivery systems. ${ }^{1-4}$ In this article we report our observations of transient pores in vesicles. Although vesicles considered in drug release formulations have small sizes of the order of $100 \mathrm{~nm}$, we have chosen to work with larger, uncoated giant unilamellar vesicles (GUVs), a few tens of micrometres in size, as model systems. Their size range makes GUVs amenable to observation by optical microscopy, which provides two major advantages: (1) vesicles can be studied individually, and (2) observations are direct and in real time.

An important parameter in these systems, effecting physical properties of lipid bilayers, is line tension. Unfortunately, however, direct measurements of line tension have been difficult in the past due to its extremely small value, of the order of $10 \mathrm{pN}$. Here we report a novel way of measuring the line tension from pore dynamics, and show how its value can be tuned over two orders of magnitude. Transient pores appear ${ }^{5,6}$ upon increasing the membrane tension of the vesicles, $\sigma$, beyond a certain value. Here, this is achieved by optical illumination, as described in Fig 1 . At the beginning of the observation, a vesicle is floppy and undergoes large amplitude transversal fluctuations since its membrane tension is nearly zero (Fig 1(a)).
Illuminating the same vesicle using the maximum available light intensity of the microscope, we observe that, little by little, the vesicle loses its fluctuations and becomes completely spherical (Fig 1(b)). This process takes between $15 \mathrm{~min}$ and a few hours, during which time the vesicle volume does not change significantly (for more details, see References 5, 7 and 8). Although the molecular mechanism of this optically induced tension build-up is not clear, it has been reported by other researchers. ${ }^{9,10}$ If we continue to illuminate the vesicle, and hence build up further tension, the vesicle finally responds by the sudden opening of a pore, as shown in Fig 2. The pore opens up very rapidly but, remarkably, after reaching a maximum radius (Fig 2(b)), it closes again, albeit very slowly (Fig 2(c)-(f)). The time evolution of the pore and vesicle radii are plotted in Fig 3 for the pore shown in Fig 2.

Pore opening is driven by the membrane tension, $\sigma$, and its closure by the line tension, $\mathcal{T}{ }^{6}$ We used viscous mixtures of glycerol and water to slow down the leaking out of the internal liquid. As will be seen shortly, this is essential for the observation of large, long-lived pores. This article is organized as follows. In the next section we present materials and methods. In a third section we summarize the hydrodynamic model which explains the pore dynamics, and allows us to

\footnotetext{
* Correspondence to: Françoise Brochard-Wyart, Institut Curie, Section de Recherche, Laboratoire PCC/UMR 168, 11, rue Pierre et Marie Curie, 75231 Paris Cedex 05, France

E-mail: brochard@curie.fr

†Oral presentation - Paper presented at the Formula III Conference: New Concepts and Strategies in Formularies, from Laboratory to Industry, 13-16 October, 2001, Hérault, France

${ }^{\ddagger}$ Current address: Erdem Karatekin, Institut de Biologie Physico-Chimique, CNRS UPR 1929, 13 rue Pierre et Marie Curie, 75005 Paris, France Contract/grant sponsor: Institut Curie

(Received 21 January 2002; revised version received 2 April 2002; accepted 18 April 2002)
} 

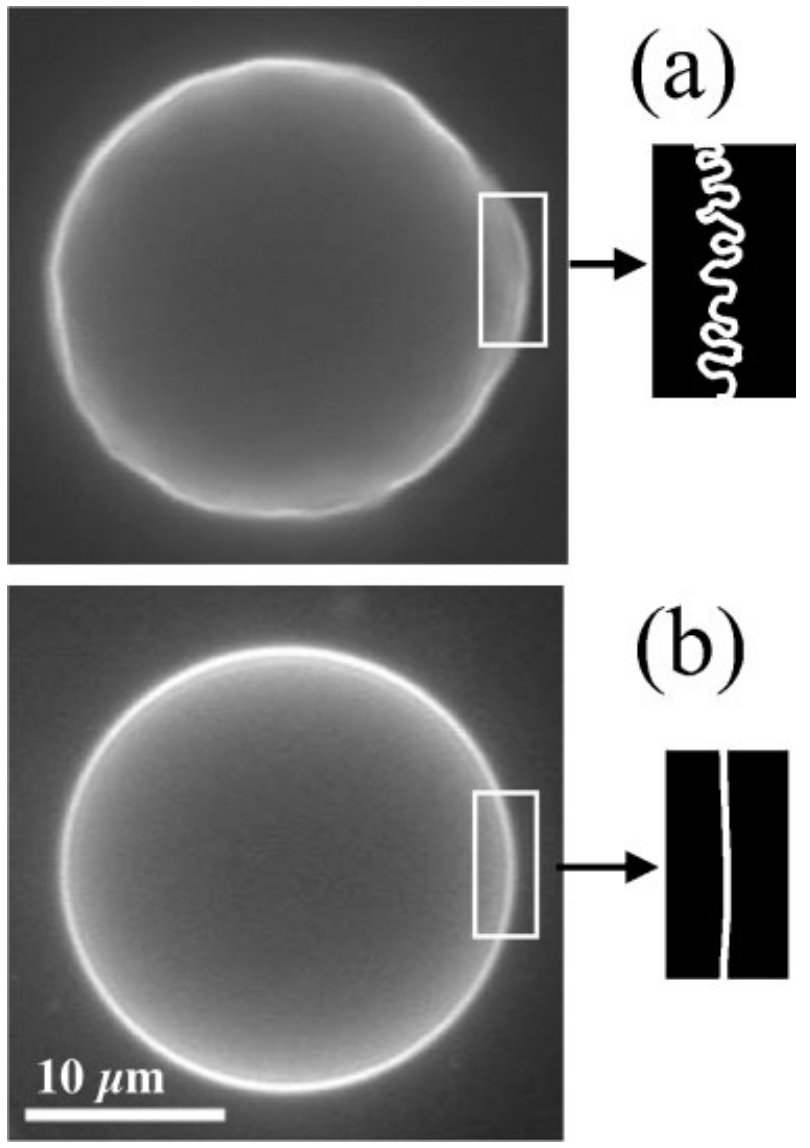

Figure 1. (a) At the beginning of the observation under a fluorescence microscope the vesicles were relaxed: their membrane tension, $\sigma$, is very small and their membrane undergoes large amplitude fluctuations. (b) Over tens of minutes of observation with maximum available incident light intensity, illuminated vesicles gradually became tense and perfectly spherical.

extract the line tension from the speed with which pores reseal. The following section is concerned with experimental results; in particular, we demonstrate how the line tension (and consequently pore lifetimes) can be tuned over two orders of magnitude. In a final section we discuss our results.

\section{EXPERIMENTAL}

Giant vesicles were prepared using the 'electroformation' method, ${ }^{11}$ which produces GUVs with high efficiency and very few multilamellar aggregates. The lipid used was 1,2-dioleoyl-sn-glycero-3-phosphocholine (DOPC, Sigma, structure 1 in Fig 4) which is in the $L_{\alpha}$ fluid phase at room temperature and forms vesicles in arbitrary mixtures of water and glycerol. ${ }^{12} \mathrm{~A}$ $1 \mathrm{mg} \mathrm{ml}^{-1}$ solution of pure lipid was cast from a $2: 1 \mathrm{v} / \mathrm{v}$ mixture of chloroform and methanol on one side each of two conducting glass plates, coated with indium tin oxide. The lipid film was dried in a vacuum oven overnight. Then the two glass plates were assembled, with the lipid-cast sides facing each other, using a $1 \mathrm{~mm}$ thick teflon spacer from which a $4 \mathrm{~cm}$ diameter circular section had been cut out. This circular section coincided with the region over which the lipid was cast. Critoseal (Prolabo, France) was used to seal around the teflon spacer. Through a gap in the spacer, about $1.5 \mathrm{ml}$ of aqueous solution $(2: 1 \mathrm{v} / \mathrm{v}$ glycerol/water, containing $100 \mathrm{mM}$ sucrose, viscosity $\eta_{o}=32 \mathrm{cP}$ ) was introduced into this electroformation cell while an AC voltage $(\sim 1.1 \mathrm{~V}, 6-8 \mathrm{~Hz})$ was applied. The formation of vesicles was monitored directly by bright field microscopy. Once the vesicles matured (in about $4-6 \mathrm{~h}$ ) the voltage was removed, and the vesicles were allowed to 'relax' in the electroformation cell overnight. For observations, the vesicles were gently transferred to a tube and labelled with lipophilic fluorescent dye n-(4-sulfopropyl)-4-(4-(dihexylamino)styryl)pyridinium ( $\mathrm{di}_{6}$ ASP-BS) (kindly provided by Mireille Blanchard-Desce, UMR 6510 CNRS, U de Rennes, France, structure 2 in Fig 4), which was spontaneously inserted into the bilayer. $1 \%$ $\mathrm{v} / \mathrm{v}$ of $560 \mu \mathrm{M}$ of the dye (in ethanol) was incubated for
Figure 2. When a tensed vesicle (a) was illuminated further, more tension built up and the vesicle finally responded by the sudden opening of a pore (b). The pore size reached its maximum very rapidly (b), thereafter decreased slowly until complete resealing (c-f). The time evolution of the pore and the vesicle radii are plotted in Fig 3. In this example, the bilayer contains $20 \mathrm{~mol} \%$ cholesterol.
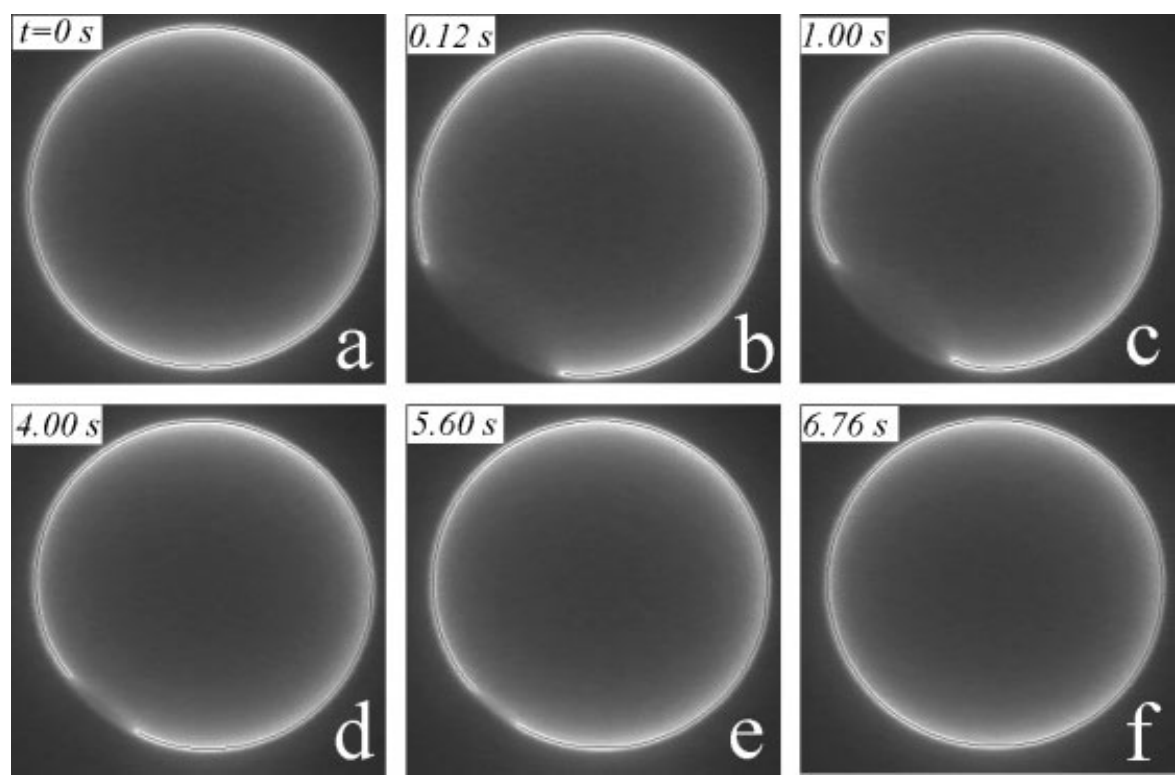

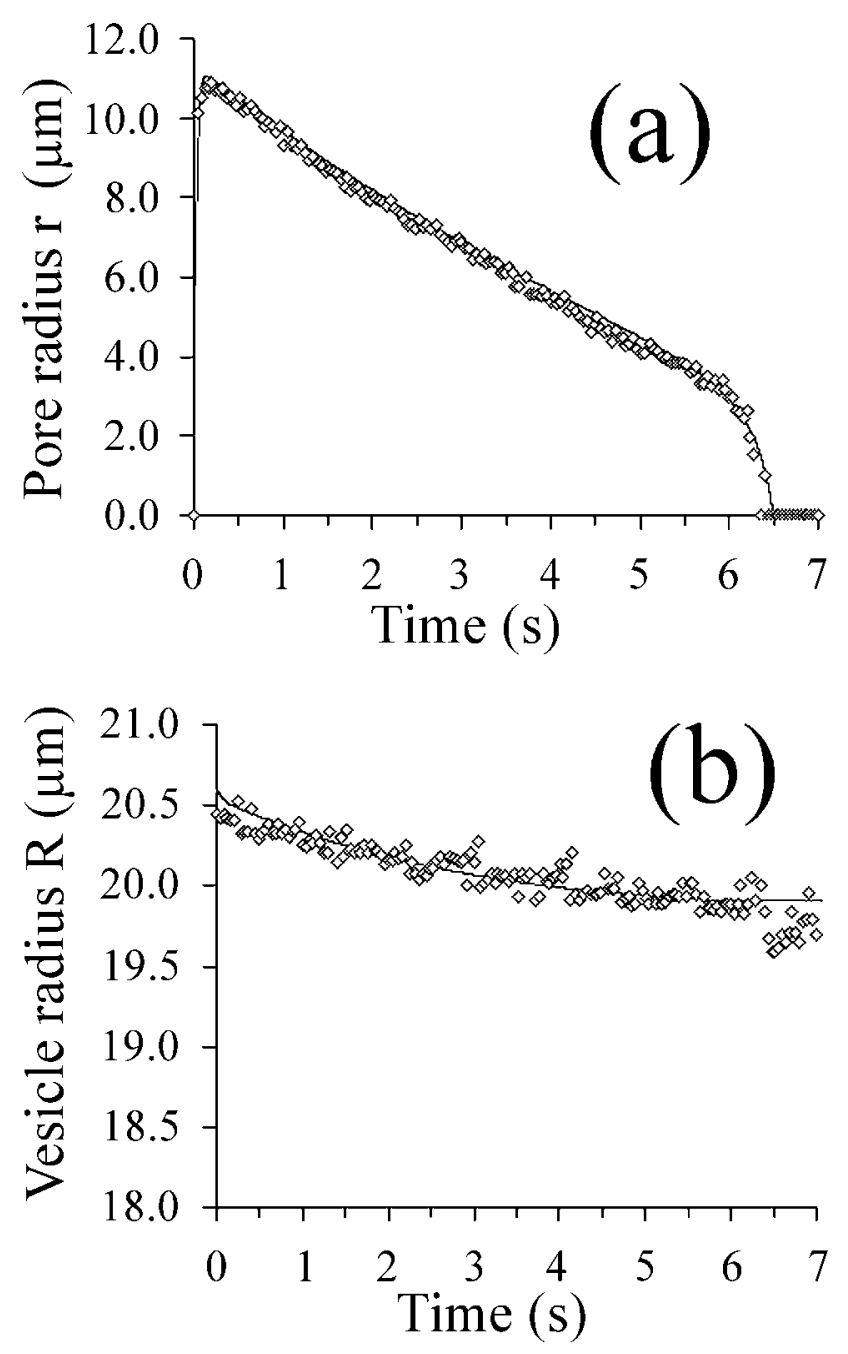

Figure 3. (a) Time evolution of the pore radius, $r$, for the pore shown in Fig 2. (b) Time evolution of the vesicle radius $R$ for the same vesicle as in Fig 2. Diamonds are experimental measurements, and the continuous curves are numerical solutions (see section III).

a few hours with the vesicle solution prior to observation. The vesicles were either observed as such, or following sedimentation in an equimolar solution, in a closed microscope chamber (two glass slides spaced by parafilm of $200 \mu \mathrm{m}$ thickness and sealed with hot paraffin), using a Reichert-Jung upright microscope equipped with an Olympus $63 \times / 0.90$ LUMPlanF1<smiles>CCCCCCCCCCCCCCCCCCCCCC(=O)OCC(C)(CO[PH]([O-])([O-])OCC[N+](C)(C)C)OC(=O)CCCCCCCCCC</smiles><smiles>O=S(=O)([O-])CCCC[n+]1ccc(/C=C/c2ccc(N(Cc3ccccc3)c3ccccc3)cc2)cc1</smiles>

Figure 4. Chemical structures of the phospholipid DOPC (1) and the fluorescent marker, $\mathrm{di}_{6} \mathrm{ASP}-\mathrm{BS}$ (2). used in the experiments. water immersion objective. Epifluorescence excitation was provided by a $200 \mathrm{~W}$ mercury lamp and a $455-$ $490 \mathrm{~nm}$ band pass filter. The optical illumination to increase the membrane tension was also provided by this lamp. The fluorescence emission of the dye at $580 \mathrm{~nm}$ was detected with a standard CCD camera. Images were digitized on an 8-bit frame grabber (LG3, Scion Corp, Frederick, MD) and analyzed using IMAGE software (National Institute of Health).

For experiments in which surfactants were added to vesicles, a stock solution of the surfactant Tween 20 (Sigma, Fig 5(c)) was prepared in aqueous solutions identical to those in which the vesicles were kept, ie $100 \mathrm{mM}$ sucrose in a $2: 1 \mathrm{v} / \mathrm{v}$ glycerol/water mixture. These stock solutions were either mixed with the vesicles prior to observation in a closed cell or were introduced through an opening in the observation cell. The latter method provided a front of surfactants diffusing into the vesicle solution, allowing the observation of the effect of surfactants on vesicles immediately. The advantage of the former method was that the concentration of the surfactant was homogeneous and well known. For the former method, we observed vesicle/surfactant solutions between $1 / 2$ and $2 \mathrm{~h}$ following mixing. The vesicles were destroyed slowly, over about 2 days, by the surfactants. With Tween 20, as well as sodium cholate (Sigma, Fig 5(b)) we were able to induce sufficient membrane tension by optical illumination to trigger the appearance of transient pores.<smiles>CC(C)CCC[C@H](C)C1CC[C@H]2[C@H]3CC=C4CC(O)CCC4(C)[C@H]3CC[C@]12C</smiles><smiles>CC(CCC(=O)O[NH3+])C1CC[C@@H]2C1C(O)CC1C2C(O)CC2CC(O)CCC21C</smiles>

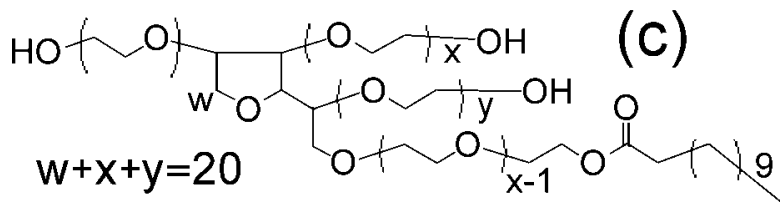

Figure 5. (a) Cholesterol, (b) sodium cholate, and (c) Tween 20. 


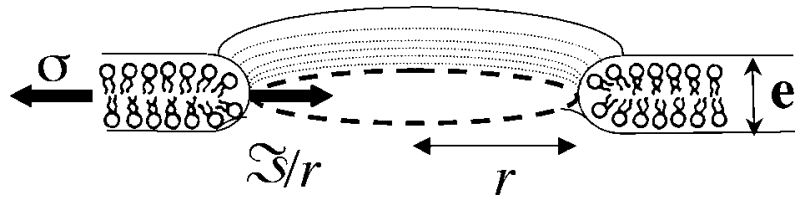

Figure 6. Transversal view of a pore, drawn schematically. Pore opening is driven by the membrane tension $\sigma$, and is opposed by the line tension term $\mathcal{T} / r$ (see text).

In experiments in which cholesterol (Fig 5(a)) was added to the bilayers, a somewhat different approach was taken since cholesterol is hydrophobic. Cholesterol was co-cast with the lipid onto glass plates, at the desired molar ratio, prior to electroformation. The rest of the procedure was similar to what is described above.

\section{HYDRODYNAMIC MODEL}

In this section we summarize the hydrodynamic model which describes the pore dynamics, following Reference 6. As can be seen in Fig 2, the overall spherical geometry of the vesicle is largely conserved during the lifetime of a pore. Experimental measurements of the pore and vesicle radii are plotted as a function of time in Fig 3 for this example. The driving force for the pore opening is the membrane tension, $\sigma$, and that for pore closure is the line tension, $\mathcal{T}$, as drawn schematically in Fig 6. The surface energy is assumed to dissipate in a viscous manner in the bilayer, which has a surface viscosity $\eta_{\mathrm{s}} \equiv e \eta_{\mathrm{m}}$, in which $\eta_{\mathrm{m}}$ is the membrane viscosity (in $\mathrm{Ns} / \mathrm{m}^{2}$ ) and $e$ is the thickness of the bilayer:

$$
2 \eta_{\mathrm{s}} \dot{r}=r \sigma-\mathcal{T} .
$$

A pore opens if its size exceeds a nucleation radius $r_{\mathrm{n}}=\mathcal{T} / \sigma_{\mathrm{o}}$, in which $\sigma_{\mathrm{o}}$ is the initial membrane tension. When the pore opens, the membrane tension $\sigma$ drops due to two factors (we assume that lipid loss during the lifetime of a pore is negligible): (1) the same amount of

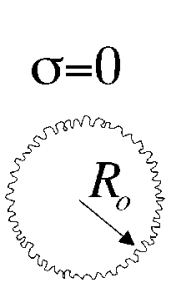

(a)

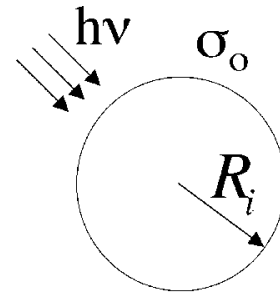

(b)

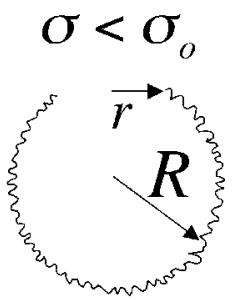

(c)
Figure 7. (a) A hypothetical, completely relaxed vesicle (membrane tension $\sigma=0$ ) with lipid area $A_{1}$ and radius $R_{0}$. (b) A 'real' vesicle with the same lipid area, but with a non-zero membrane tension $\sigma_{0}$ has a projected area $A_{\mathrm{p}}$ that is $\left(1+\sigma_{\mathrm{o}} / E\right)$ times larger. (c) As a pore opens, the membrane tension drops. The vesicle radius, $R$, also decreases somewhat, due to leaking out of the inner liquid. lipid is now distributed over a surface area that is smaller by the area of the pore, allowing the membrane to 'wrinkle', which relaxes $\sigma$ (cf Figs 7(b) and (c)), and (2) the internal liquid leaks out due to the excess laplace pressure, $\Delta P=2 \sigma / R$, present in the vesicle (Fig $8)$. As the liquid leaks out, the volume of the vesicle (and its radius $R$ ) decreases. We now derive an expression describing how $\sigma$ varies as a function of the pore $(r)$ and vesicle $(R)$ radii, taking into account (1) and (2) above, by counting areas, as follows. As shown in Fig 7(b), just prior to the opening of a pore, the vesicle has a membrane tension $\sigma_{\mathrm{o}}$, radius $R_{\mathrm{i}}$, and an area $4 \pi R_{\mathrm{i}}^{2}$. The same lipid area can be expressed in terms a radius $R_{\mathrm{o}}$ that the vesicle would adopt were its membrane tension $\sigma=0$ (this hypothetical situation is drawn schematically in Fig $7(\mathrm{a})): 4 \pi R_{\mathrm{i}}^{2}=4 \pi R_{\mathrm{o}}^{2}$ $\left(1+\sigma_{\mathrm{o}} / E\right)$, in which $E$ is a two dimensional elastic modulus, associated with the undoing of the wrinkles. ${ }^{13}$ In other words, a non-zero membrane tension $\sigma_{\mathrm{o}}$ stretches a vesicle by a factor $\left(1+\sigma_{\mathrm{o}} / E\right)$ (cf Figs 7 (a) and (b)). Now let us assume for the moment that the contents of the vesicle are gelified and that no leak out occurs as a pore opens. The total area count then becomes $4 \pi R_{\mathrm{i}}^{2}=4 \pi R_{\mathrm{o}}^{2}\left(1+\sigma_{\mathrm{o}} / E\right)=4 \pi R_{\mathrm{o}}^{2}$ $(1+\sigma / E)+\pi r^{2}$ after replacing $\sigma_{\mathrm{o}} \rightarrow \sigma$ and accounting for the area of the pore. From this, we define a critical radius, $r_{\mathrm{c}} \equiv 2 R_{\mathrm{o}} \sqrt{\sigma_{\mathrm{o}} / E}$, to which the pore size would need to grow to relax the membrane tension completely, ie $\sigma=0$. In the general case where leak out is permitted, we replace $R_{\mathrm{i}} \rightarrow R$ and rearrange the above

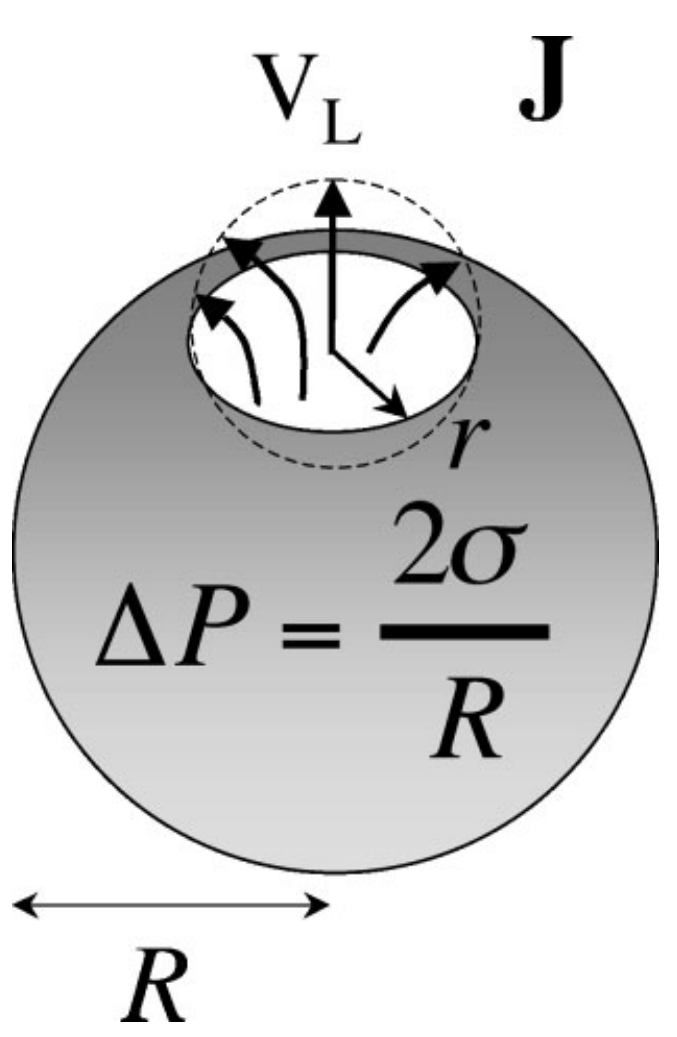

Figure 8. The inner liquid of a tensed vesicle leaks out in the presence of a pore (radius $r$ ), due to the excess Laplace pressure $\Delta P=2 \sigma / R$. The flux through the pore is $\mathrm{J}$, and the velocity of the outflowing liquid is $V_{\mathrm{L}}$ at the centre of the pore. 
expression for the area count to obtain:

$$
\frac{\sigma}{\sigma_{\mathrm{o}}}=1-\frac{r^{2}}{r_{\mathrm{c}}^{2}}-\frac{R_{\mathrm{i}}^{2}-R^{2}}{R_{\mathrm{i}}^{2}-R_{\mathrm{o}}^{2}}, \quad r_{\mathrm{c}} \equiv 2 R_{\mathrm{o}}\left(\frac{\sigma_{\mathrm{o}}}{E}\right)^{1 / 2}
$$

It remains to describe how the vesicle radius, $R$, in Eqn (2) changes during the lifetime of a pore. For this, we consider the flux through the pore which can be thought of as a circular orifice of radius $r$, across which the pressure drop is $\Delta P$ (see eg Reference 14). The pressure drop is given by the laplace pressure $\Delta P=2 \sigma / R$. Equating the flux to the rate of change of the vesicle volume, we find:

$$
-4 \pi R^{2} \dot{R}=\frac{2 \sigma}{3 \eta_{\mathrm{o}} R} r^{3}
$$

Strictly speaking, eqn (3) only applies to flow through an orifice with constant radius. However, it is a good approximation for our problem as long as $r$ changes slowly: ${ }^{6} \dot{r} \lesssim V_{\mathrm{L}} \approx \Delta P r / \eta_{\mathrm{O}}$, in which $V_{\mathrm{L}}$ is the velocity of liquid flowing through the centre of the pore.

Equations (1), (2) and (3) describe the dynamics of a pore completely. Numerical solutions of these equations (solid curves) are fitted to the data in Fig 3 for $r(t)$ and $R(t)$. As discussed in Ref 6, when eqns (1), (2) and (3) are rewritten using the reduced variables $\tilde{r} \equiv r / r_{\mathrm{c}}, \Delta \equiv\left(R_{\mathrm{i}}-R\right) /\left(R_{\mathrm{i}}-R_{o}\right), \tilde{\sigma} \equiv \sigma / \sigma_{\mathrm{o}}$, and $\tilde{t} \equiv t / \tau$, where $\tau \equiv 2 \eta_{\mathrm{s}} / \sigma_{\mathrm{o}}$, a parameter, $\tilde{r_{\mathrm{L}}}$, related to the efficiency of leak out, readily emerges:

$$
\tilde{r}_{\mathrm{L}} \equiv \frac{3 \pi \eta_{\mathrm{o}}}{8 \eta_{\mathrm{s}}} \frac{R_{\mathrm{o}}^{2}}{r_{\mathrm{c}}} \quad \text { (leak-out parameter) }
$$

Since this parameter depends on the viscosity of the inner liquid, $\eta_{\mathrm{o}}$, it can be tuned experimentally. When it is small $\left(\tilde{r_{\mathrm{L}}} \ll 1\right)$, as in water, the pores are aborted before they grow to any appreciable sizes. This is because, as soon as a pore opens, leak-out provides an efficient way to relax the membrane tension $\sigma$. No longer having a strong driving force for opening, the pores reseal quickly due to the line tension.
In contrast, when the 'leak-out parameter', $\tilde{r_{L}}$, is made large $\left(\tilde{r_{L}} \gg 1\right)$ by use of mixtures of glycerol and water, pores open almost to their critical size, $r_{c}$, because leak-out is slowed down. Leak-out is much slower than the pore opening, which takes time $\tau \equiv 2 \eta_{\mathrm{s}} / \sigma_{\mathrm{o}}$, and so the opening occurs as if the contents of the vesicle were gelified, up to a maximum value ${ }^{6}$ $r_{\mathrm{m}} \approx r_{\mathrm{c}}-r_{\mathrm{n}} / 2 \approx r_{\mathrm{c}}$. At that point we have a situation of quasi-equilibrium between the opening $(r \sigma)$ and the closing $(\mathcal{T})$ forces:

$$
r \sigma \approx \mathcal{T}
$$

which is maintained for some time as the contents leak out very slowly. This slow closure regime is experimentally the most relevant one, as it lasts longest (approximately from $0.1 \mathrm{~s}$ to $6 \mathrm{~s}$ in Fig 3) and it is the regime which allows us to measure line tensions, as explained below. During this regime, another quantity which changes very slowly is the membrane tension $\sigma$. Thus, we set $\dot{\sigma}=0$ to obtain $4 R \dot{R}=r \dot{r}$, from eqn. (2). This yields, after use of eqns (3) and (5), the closure velocity during the slow closure regime:

$$
\dot{r} \approx \frac{-2}{3 \pi} \frac{\mathcal{T}}{\eta_{\mathrm{o}}} \frac{r}{R^{2}}
$$

which, remarkably, is independent of the surface viscosity $\eta_{\mathrm{s}}$. The only unknown in eqn (6) is the line tension $\mathcal{T}$; measuring the closure velocity in this regime allows us to infer $\mathcal{T}$. In practice, to infer $\mathcal{T}$ we use the following method described in detail in Ref 8. Integrating eqn (6) with the approximation $R \approx$ constant, we obtain $R^{2} \ln r \approx-2 \mathcal{T} /\left(3 \pi \eta_{\mathrm{o}}\right) t+C$, where $C$ is a numerical constant. That $R$ changes little over the course of the pore lifetime is apparent in Fig 3(b). Plotting the quantity $R^{2} \ln r$ against $t$ yields a straight line whose measured slope, $-\left(2 \mathcal{T} / 3 \pi \eta_{\mathrm{o}}\right)$, is used to obtain $\mathcal{T}$, as illustrated in Fig 9 .
Figure 9. $R^{2} \ln (r)$ versus $t$ for the pore shown in Fig 2. The straight line portion of this plot has slope $-\left(2 \mathcal{T} / 3 \pi \eta_{0}\right)$, from which $\mathcal{T}$ is calculated to be $13 \mathrm{pN}$ for this bilayer, containing $20 \mathrm{~mol} \%$ cholesterol.

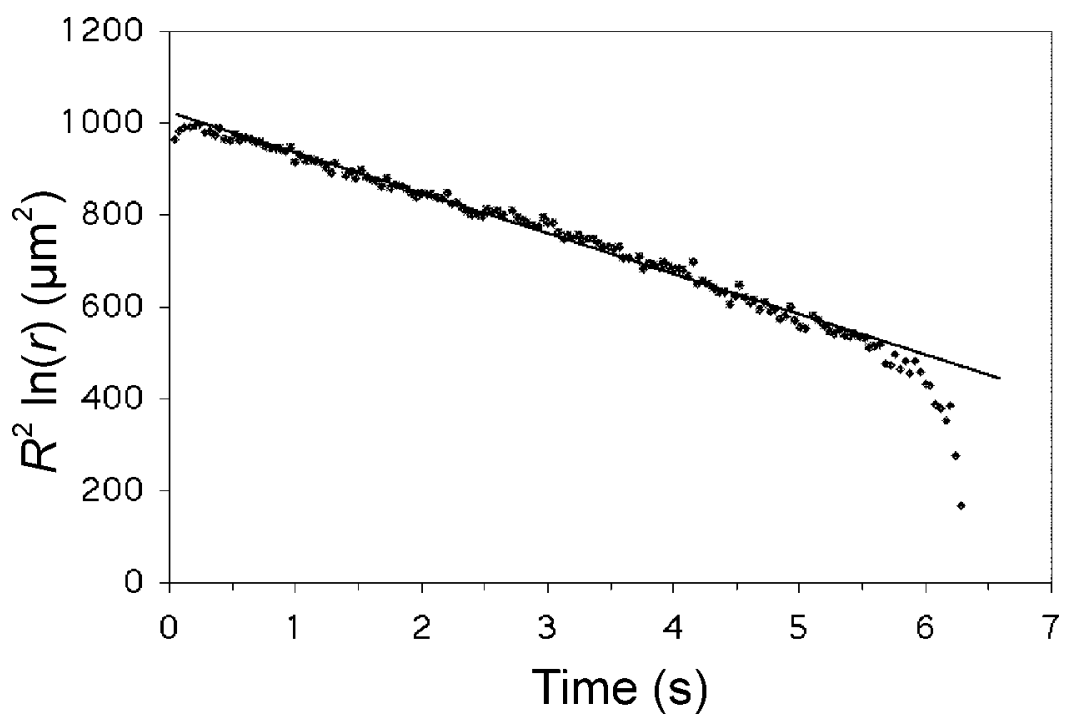



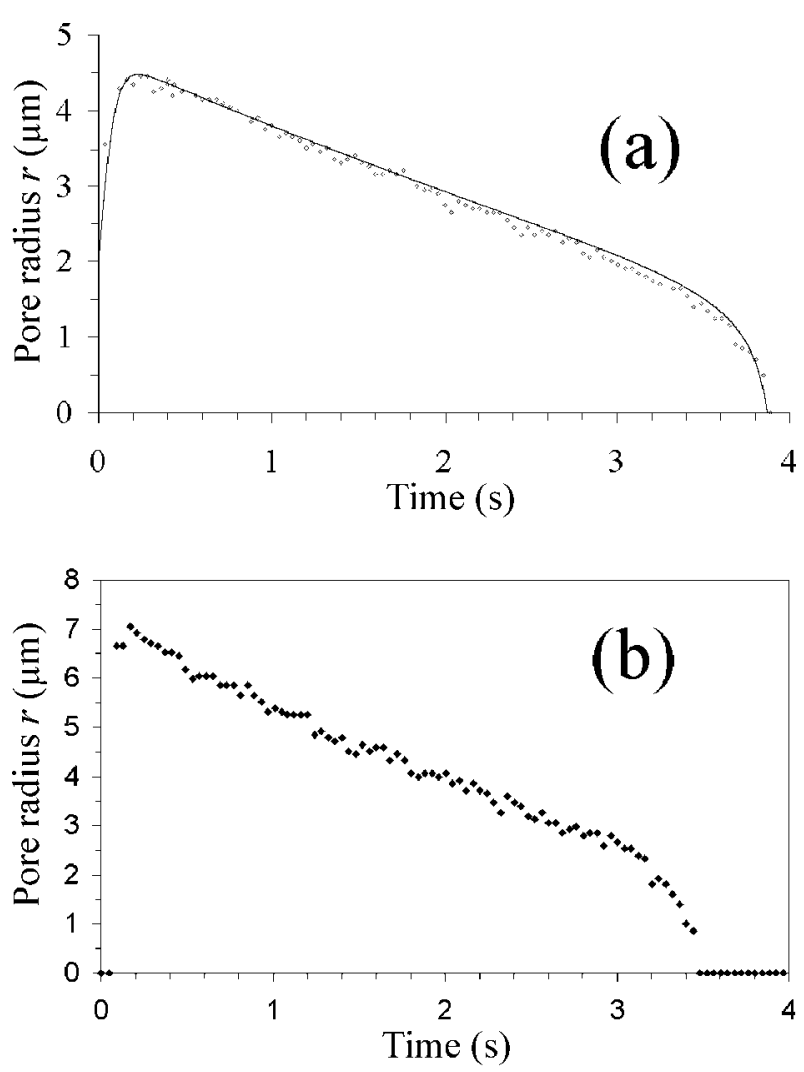

Figure 10. Pore dynamics in the absence (a) and presence (b) of cholesterol. (a) Pore radius as a function of time for a bare DOPC vesicle, with $R_{0} \approx 14 \mu \mathrm{m}$. (b) Same as in (a), but for a vesicle with $20 \mathrm{~mol} \%$ cholesterol, and $R_{\mathrm{o}} \approx 16 \mu \mathrm{m}$.

\section{RESULTS: TUNING PORE LIFETIMES}

The ability to tune the size and the lifetime of the pores could be very useful for eventual formulations in controlled drug release applications using vesicles as carriers of active substances. One of the ways of varying pore lifetimes, ${ }^{6}$

$$
t_{\text {pore }} \approx(3 \pi / 2)\left(\eta_{\mathrm{o}} R_{\mathrm{o}}^{2} / \mathcal{T}\right)
$$

is by modifying the line tension $\mathcal{T}$. First, we attempted to reduce pore lifetimes by increasing $\mathcal{T}$ by addition of cholesterol (Fig 5(a)) to DOPC vesicles. As shown in Fig 10, pore lifetimes are shortened compared with pores observed in bare DOPC vesicles of comparable size. For a bare DOPC vesicle with a radius $R_{o} \approx 14 \mu \mathrm{m}$, the pore lifetime (Fig $10(\mathrm{a})$ ), is longer than that of a slightly larger vesicle $\left(R_{o} \approx 16 \mu \mathrm{m}\right)$ containing $20 \mathrm{~mol} \%$ cholesterol (Fig 10(b)). If the line tensions were the same for both vesicles, the pore lifetime of the larger vesicle should be longer by a factor of $(16 \mu \mathrm{m})^{2} /(14 \mu \mathrm{m})^{2} \approx 1.3$.

In Fig 11 we calculate the line tension for these two cases from the slopes of plots of $R^{2} \ln r$ against $t$. As shown in Fig $11(\mathrm{a}), \mathcal{T} \approx 8.1 \mathrm{pN}$ is found for bare DOPC (pore in Fig 10(a)). However, for bilayers containing $20 \mathrm{~mol} \%$ cholesterol (pore in Fig 10(b)), we find $\mathcal{T} \approx 12.6 \mathrm{pN}$, from the plot in Fig $11(\mathrm{~b})$. Thus, addition of cholesterol indeed reduces pore lifetimes (for vesicles of comparable size) by increasing the line tension. Carrying out similar measurements in the presence of different concentrations of cholesterol we find an approximately linear increase in $\mathcal{T}$ as a function of cholesterol content, at least up to about $20-30 \%$ cholesterol. $^{7,8}$

Next, we tried to increase pore lifetimes by reducing the line tension by addition of surfactants. Effects of some surfactants on liposomes in low viscosity solutions have recently been visualized by Nomura and coworkers ${ }^{15}$ using dark-field microscopy. As shown in Fig 12, in the presence of the surfactant Tween 20, the pore lifetime for a vesicle of size $R_{\mathrm{o}} \approx 10 \mu \mathrm{m}$ is longer than $2 \mathrm{~min}$. The line tension for this pore is estimated to be $\mathcal{T} \approx 0.2 \mathrm{pN}$, from a plot of $R^{2} \ln r$ against $t$, as shown in Fig 13. In that particular experiment, a concentration gradient was used, across which the concentration of the surfactant varied from zero to $\approx 0.6 \mathrm{mM}$ (about $10 \times$ the CMC). With another surfactant, sodium cholate (Fig 5(b)), we observed similar increases in pore lifetimes. We are currently exploring systematically the effect of surfactant concentration on the line tension with several different surfactants. These will be reported in a forthcoming article. $^{8}$
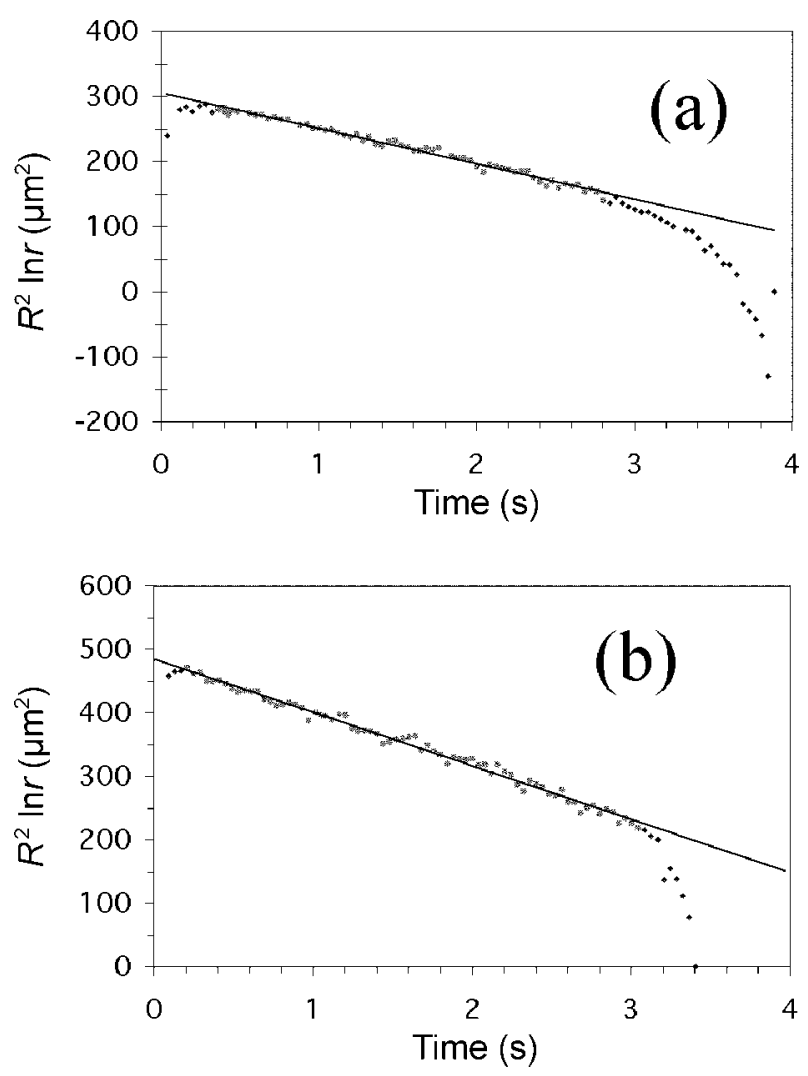

Figure 11. Calculation of the line tension in the absence (a) and presence (b) of cholesterol. (a) $R^{2} \ln r$ as a function of time, for the pore in Fig 10(a). The linear portion of the plot has slope $-54.3 \mu \mathrm{m}^{2} \mathrm{~s}^{-1}$, which implies $\mathcal{T} \approx 8.1 \mathrm{pN}$. (b) As in (a), but for the pore in Fig 10(b), for a membrane containing $20 \mathrm{~mol} \%$ cholesterol. The slope of the least squares fit to the linear region is $-84.2 \mu \mathrm{m}^{2} \mathrm{~s}^{-1}$, which yields $\mathcal{T} \approx 12.6 \mathrm{pN}$. 


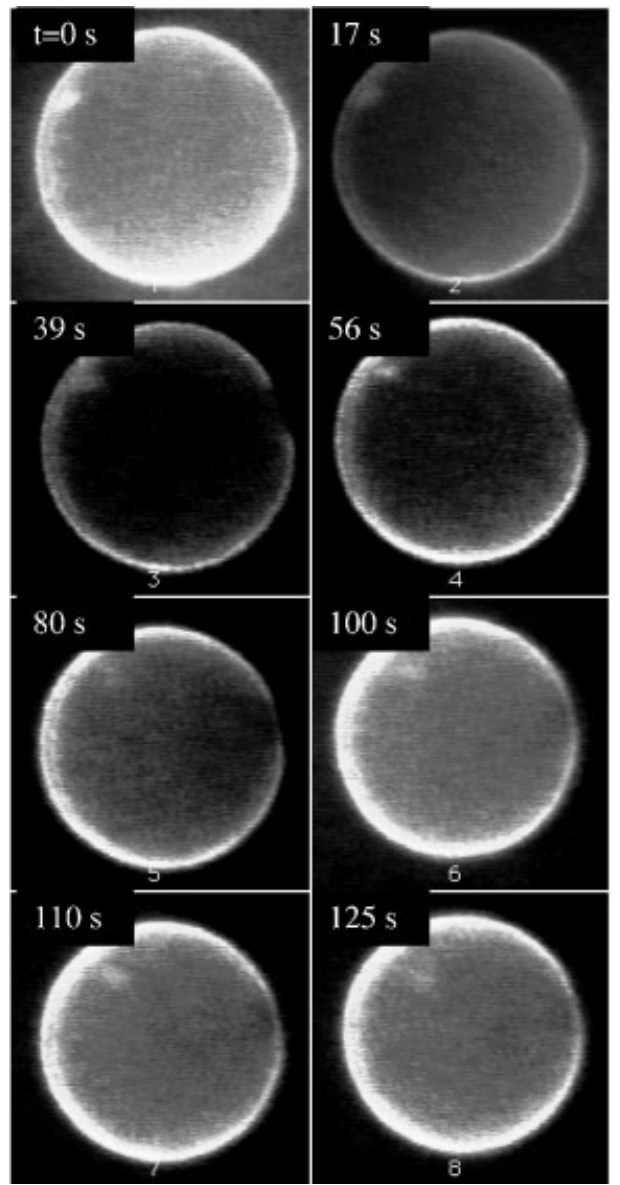

Figure 12. A long-lived pore in the presence of the surfactant Tween 20 $\left(R_{\mathrm{o}} \approx 10 \mu \mathrm{m}\right)$.

While studying the effect of surfactants on pore lifetimes, we have noticed that fusion between vesicles are greatly facilitated in the presence of either Tween 20 or sodium cholate. Fusion appears to proceed very rapidly through the opening of a pore between the two fusing vesicles, as shown in Fig 14. The time between the moment the two vesicles touch (frame $3,0.80 \mathrm{~s}$ in Fig 14) till the opening of the 'fusion pore' (frame 5,

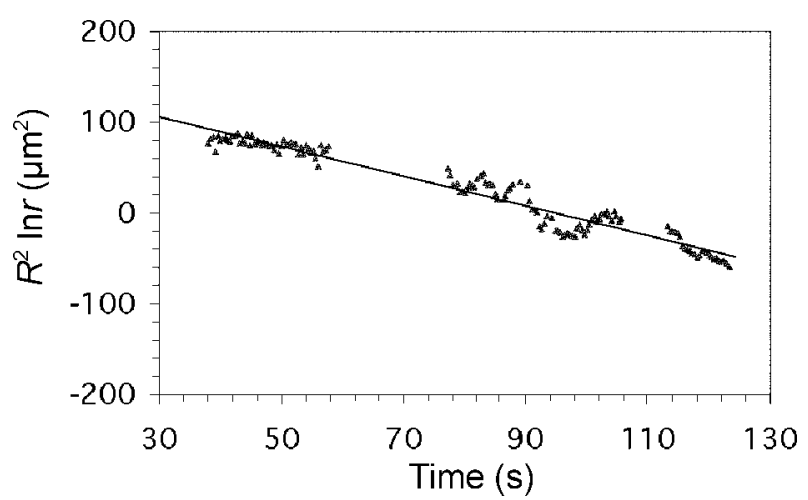

Figure 13. The linear portion of a plot of $R^{2} \ln r$ against $t$ for the pore shown in Fig 12, in the presence of the surfactant Tween 20. From this plot $\mathcal{T} \approx 0.2 \mathrm{pN}$ is found $\left(R_{\mathrm{o}} \approx 10 \mu \mathrm{m}\right)$.
$0.88 \mathrm{~s}$ in Fig 14 ) is only about $0.1 \mathrm{~s}$. In sharp contrast, fusion events are rarely seen for bare DOPC vesicles.

\section{DISCUSSION}

We have presented our observations of transient pores in giant unilamellar vesicles placed under tension. Although in this study all the vesicles were stretched by optical means, there are other, better understood (but not always as convenient) ways of inducing membrane tension, such as using micropipettes, ${ }^{16}$ or adsorption of vesicles onto appropriately modified surfaces, ${ }^{5}$ or on colloidal particles. ${ }^{8}$

We have shown how the line tension of the membranes could be modified in a controlled manner over nearly two orders of magnitude, by addition of cholesterol or surfactants to the bilayers. Since pore lifetimes are inversely proportional to the line tension, these can be tuned accordingly. The ability to control pore lifetimes would be highly desirable in potential formulations of liposomal drug delivery systems.

We have also shown that the vesicles need to be prepared in viscous solutions in order to obtain large, long-lived pores that can be visualized. What happens in the biologically more relevant case of aqueous solutions which are 30 times less viscous than the ones we have used? When we attempt to extrapolate our results to water, we speculate that in water pores would also appear in tensed vesicles, but would be much smaller and shorter-lived, and thus difficult to visualize. This is consistent with the fact that we have so far not been able to observe pores directly in water. However, the presence of pores in such systems have been suggested by indirect measurements: anomalously high permeation rates, explained by the presence of small, transient pores, have been observed for tensed vesicles in water., ${ }^{5,17,18}$ Other researchers have been able to visualize long-lived, large pores in low viscosity solutions by creating the pores by electroporation, and maintaining large membrane tensions using micropipette aspiration. ${ }^{16,19}$ If the driving force for pore opening, the membrane tension, is allowed to relax by leak out, as is the case in our experiments, pores would be aborted before reaching observable sizes.

Finally, we have shown that the presence of surfactants appears to greatly facilitate fusion events between vesicles, presumably by lowering the energy barrier to open a fusion pore. There is close analogy between fusing vesicles and coalescing emulsion droplets. The latter problem is addressed in Refs 20 and 21 . We are currently studying the effects of surfactant concentration and spontaneous curvature on line tension and vesicle fusion.

\section{ACKNOWLEDGEMENTS}

We thank Pierre-Henri Puech, Nicolas Borghi and Hicham Guitouni for stimulating discussions. EK 


\section{$10 \mu \mathrm{m}$}

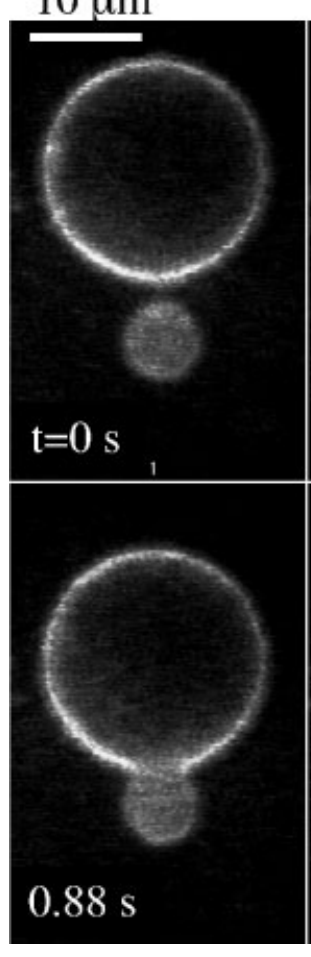

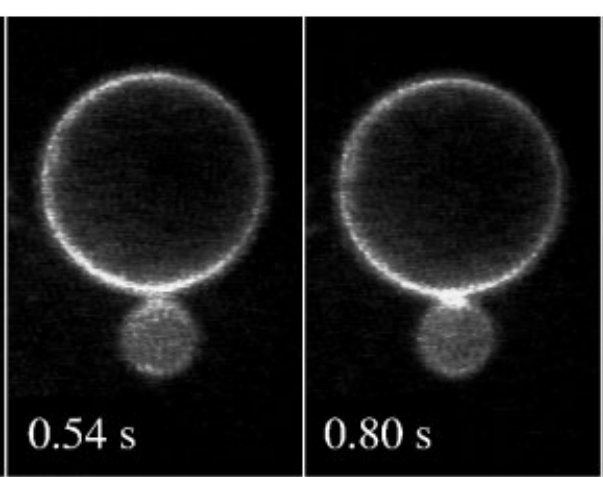
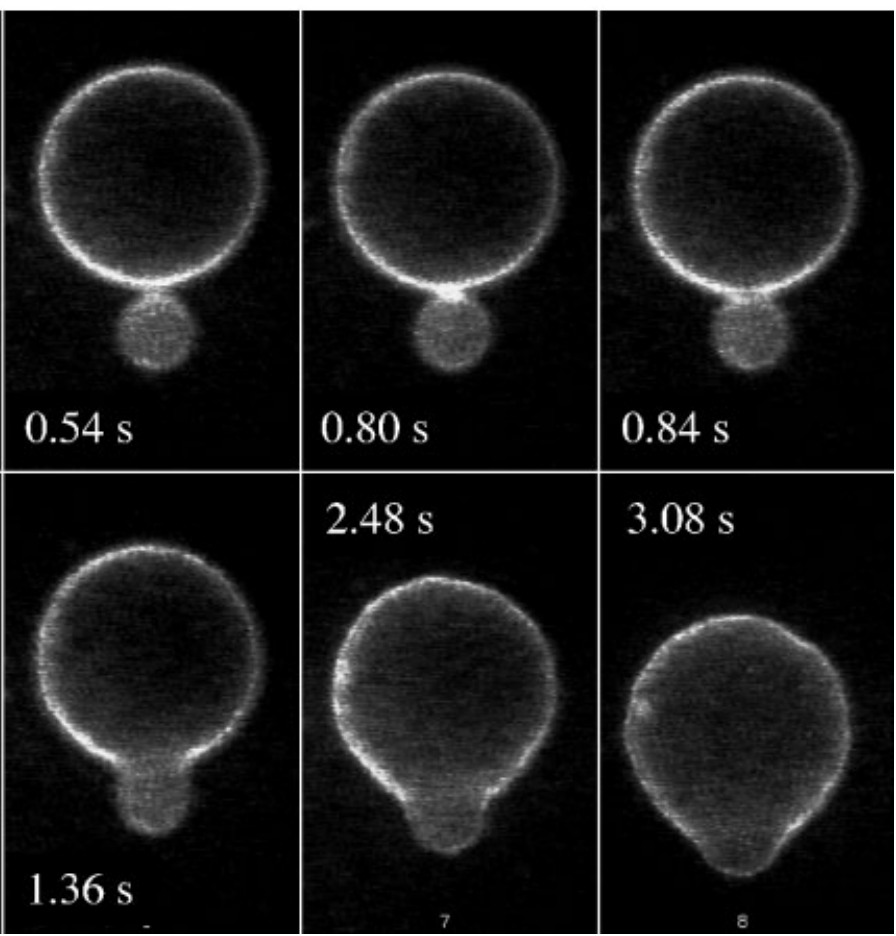

the presence of Tween 20

gratefully acknowledges an Institut Curie postdoctoral fellowship, and support by ARC and FRM.

\section{REFERENCES}

1 Lasic DD and Needham D, The 'Stealth' liposome: a prototypical biomaterial. Chem Rev 95:2601-2628 (1995).

2 Moase EH, Qu W, Ishida T, Gabos Z, Longenecker BM, Zimmermann GL, Ding L, Krantz $M$ and Allen TM, AntiMUC-1 immunoliposomal doxorubicin in the treatment of murine models of metastatic breast cancer. BBA-Biomembranes 1510:43-55 (2001).

3 Cao Y and Suresh MR, Bispecific MAb aided liposomal drug delivery. F Drug Targeting 8:257-266 (2000).

4 Yu RZ, Geary RS, Leeds JM, Watanabe T, Fitchett JR, Matson JE, Metha R, Hardee GR, Templin MV, Huang K, Newman MS, Quinn Y, Uster P, Zhu G, Working PK, Horner M, Nelson J and Levin AA, Pharmacokinetics and tissue disposition in monkeys of an antisense oligonucleotide inhibitor of Ha-ras encapsulated in stealth liposomes. Pharmaceutical Research 16:1309-1315 (1999).

5 Sandre O, Moreaux L and Brochard-Wyart F, Dynamics of transient pores in stretched vesicles. Proc Natl Acad Sci USA 96:10591-10596 (1999).

6 Brochard-Wyart F, de Gennes PG and Sandre O, Transient pores in stretched vesicles: role of leak-out. Physica A 278:3251 (2000).

7 Sandre O, Pores transitoires, adhésion et fusion des vésicules géeantes, PhD thesis, Université Paris 6, Paris, France (2000).

8 Karatekin E, Sandre O, Guitouni H, Borghi N, Puech P-H and Brochard-Wyart F, Cascades of Transient Pores in Giant Vesicles: Line Tension and Transport. Biophys $\mathcal{F}$, in press.

9 Bar-Ziv R, Frisch T and Moses E, Entropic expulsion in vesicles. Phys Rev Lett 75:3481-3484 (1995).

10 Bar-Ziv R, Nelson P and Moses E, Dynamic excitations in membranes induced by optical tweezers. Biophys f 75:294-320 (1998).

11 Angelova MI, Liposome electroformation, in Giant Vesicles, ed by Luisi PL and Walde P John Wiley, Chichester, pp 27-36 (2000).

12 Öradd G, Wikander G, Lindblom G, Johansson LB- Ä, Effect of glycerol on the translational and rotational motions in lipid bilayers studied by pulsed-field gradient ${ }^{1} \mathrm{Hnmr}$, epr and timeresolved fluorescence spectroscopy. 7 Chem Soc Faraday Trans 90:305-309 (1994).

13 Evans E and Rawics W, Entropy-driven tension and bending elasticity in condensed-fluid membranes. Phys Rev Lett 64:2094-2097 (1990).

14 Happel J and Brenner $\mathrm{H}$, in Axisymmetrical flow in low Reynolds number hydrodynamics, ed by Nijhoff $M$ Kluwer, The Hague, pp 97-158 (1983).

15 Nomura $F$, Nagata $M$, Inaba $T$, Hiramatsu $H$, Hotani $H$ and Takiguchi K, Capabilities of liposomes for topological transformation. Proc Natl Acad Sci USA 98:2340-2345 (2001).

16 Zhelev DV and Needham D, Tension-stabilized pores in giant vesicles: determination of pore size and pore line tension. Biochim Biophys Acta 1147:89-104 (1993).

17 Guedeau-Boudeville M-A, Jullien L and di Meglio J-M, Drug delivery: piercing vesicles by their adsorption onto a porous medium. Proc Natl Acad Sci USA 92:9590-9592 (1995).

18 Bernard A-L, Guedeau-Boudeville M-A, Sandre O, Palacin S, di Meglio J-M and Jullien L, Permeation through lipid bilayers by adhesion of giant vesicles on decorated surfaces. Langmuir 16:6801-6808 (2000).

19 Moroz JD and Nelson P, Dynamically stabilized pores in bilayer membranes. Biophys f 72:2211-2216 (1997).

20 Babak VG and Stebe M-J, A review on highly concentrated emulsions: physio-chemical principles of formulation. $F D S T$ 23:1-22 (2002).

21 Kabalnov A and Wennerström H, Macroemulsion stability: the oriented wedge theory revisited. Langmuir 12:276-292 (1996). 